\title{
Genome-Wide DNA Methylation Patterns in Naive CD4+ T Cells From Patients With Primary Sjögren's Syndrome
}

\author{
Nezam Altorok, ${ }^{1}$ Patrick Coit, ${ }^{2}$ Travis Hughes, ${ }^{2}$ Kristi A. Koelsch, ${ }^{3}$ Donald U. Stone, ${ }^{4}$ \\ Astrid Rasmussen, ${ }^{3}$ Lida Radfar, ${ }^{4}$ R. Hal Scofield, ${ }^{5}$ Kathy L. Sivils, ${ }^{3}$ \\ A. Darise Farris, ${ }^{3}$ and Amr H. Sawalha ${ }^{2}$
}

\begin{abstract}
Objective. Primary Sjögren's syndrome (SS) is a systemic autoimmune disease with incompletely understood etiology. This study was undertaken to investigate the role of epigenetic dysregulation in the pathogenesis of primary SS.

Methods. A genome-wide DNA methylation study was performed in naive CD4+ $\mathrm{T}$ cells from 11 patients with primary SS compared to age-, sex-, and ethnicitymatched healthy controls. Cytosine methylation was quantified using the Illumina Infinium HumanMethylation 450 BeadChip array, and the data were validated using bisulfite sequencing.

Results. Genome-wide analyses identified 553 hypomethylated CpG sites and 200 hypermethylated CpG sites in naive CD4+ $\mathrm{T}$ cells from patients with primary SS as compared to healthy controls, representing 311 hypomethylated and 115 hypermethylated gene regions. The hypomethylated genes in patients with primary SS included $L T A$ (encoding lymphotoxin $\alpha$ ). Other relevant
\end{abstract}

Supported by the NIH (National Institute of Allergy and Infectious Diseases grant R01-AI-097134 and National Institute for Arthritis and Musculoskeletal and Skin Diseases grant P50-AR060804) and the Lupus Research Institute.

${ }^{1}$ Nezam Altorok, MD: University of Michigan, Ann Arbor, and University of Toledo Medical Center, Toledo, Ohio; ${ }^{2}$ Patrick Coit, BS, Travis Hughes, MPH, Amr H. Sawalha, MD: University of Michigan, Ann Arbor; ${ }^{3}$ Kristi A. Koelsch, PhD, Astrid Rasmussen, $\mathrm{MD}, \mathrm{PhD}$, Kathy L. Sivils, PhD, A. Darise Farris, PhD: Oklahoma Medical Research Foundation, Oklahoma City; ${ }^{4}$ Donald U. Stone, MD, Lida Radfar, DDS: University of Oklahoma Health Sciences Center, Oklahoma City; ${ }^{5}$ R. Hal Scofield, MD: Oklahoma Medical Research Foundation and University of Oklahoma Health Sciences Center, Oklahoma City.

Dr. Scofield has received consulting fees, speaking fees, and/or honoraria from Lilly (less than $\$ 10,000$ ).

Address correspondence to Amr H. Sawalha, MD, Division of Rheumatology, Department of Internal Medicine, University of Michigan, 5520 MSRB-1, SPC 5680, 1150 West Medical Center Drive, Ann Arbor, MI 48109. E-mail: asawalha@umich.edu.

Submitted for publication July 16, 2013; accepted in revised form October 31, 2013. genes, such as CD247, TNFRSF25, PTPRC, GSTM1, and PDCD1, were also hypomethylated. The interferon signature pathway was represented by hypomethylation of STAT1, IFI44L, USP18, and IFITM1. A group of genes encoding members of the solute carrier proteins were differentially methylated. In addition, the transcription factor gene $R U N X 1$ was hypermethylated in patients with primary SS, suggesting a possible connection to lymphoma predisposition. Gene ontology (GO) analysis of hypomethylated genes demonstrated enrichment of genes involved in lymphocyte activation and immune response. GO terms for hypermethylated genes included antigen processing and presentation.

Conclusion. This is the first epigenome-wide DNA methylation study in patients with primary SS. These findings highlight a role for DNA methylation in primary SS and identify disease-associated DNA methylation changes in several genes and pathways in naive CD4+ $T$ cells from patients with primary SS that may be involved in the pathogenesis of this disease.

Primary Sjögren's syndrome (SS) is a complex autoimmune disease characterized by production of autoantibodies against RNP particles (Ro/SSA and La/ SSB) and muscarinic acetylcholine receptor antigens, dysfunction of water transport processes, and lymphocytic infiltration of exocrine glands resulting in glandular atrophy and dysfunction (1,2). Although xerostomia (dry mouth) and xerophthalmia (dry eyes) are the main clinical features of primary SS (3), the full spectrum of the disease encompasses involvement of different organ systems and predisposition to lymphoproliferative disease (4). Primary SS predominantly affects women, with a female-to-male distribution of 9 to 1 (5).

The etiology of primary SS is incompletely understood, but a growing body of evidence suggests that diseases with certain clinical features similar to those of 
primary SS, such as systemic lupus erythematosus (SLE), have common epigenetic factors that may contribute to the pathogenesis of autoimmunity (6). Furthermore, recent evidence suggests that global DNA methylation is reduced in salivary gland epithelial cells from patients with primary SS (7). DNA methylation is considered the core epigenetic mechanism that regulates gene expression, by altering transcriptional accessibility of regulatory regions within gene sequences (8). In a recent study, we characterized the DNA methylation changes in naive $\mathrm{CD} 4+\mathrm{T}$ cells from patients with lupus, revealing that changes in DNA methylation occurred prior to the differentiation and activation of $\mathrm{T}$ cells, and demonstrating that interferon (IFN)-regulated genes in naive $\mathrm{CD} 4+\mathrm{T}$ cells from patients with lupus are epigenetically poised for transcription (9). In the present study, we performed a genome-wide DNA methylation study in naive CD4 $+\mathrm{T}$ cells from patients with primary SS and healthy control subjects. We also validated our results using bisulfite sequencing of selected differentially methylated loci. The findings demonstrated a pattern of differential methylation in key genes and disease-associated pathways pertinent to the pathogenesis of primary SS.

\section{PATIENTS AND METHODS}

Patients and controls. We studied 11 patients with primary SS and 11 healthy control subjects. Patients and controls were matched for age (within 5 years), sex, and ethnicity. The demographic features and medications of the study participants are shown in Table 1. Classification of primary SS was based on the American-European Consensus Group 2002 revised criteria (10). The clinical features of the patients with primary SS included in this study are shown in Table 2. Disease manifestations in the patients were extensively characterized by a team of oral, ocular, and rheumatology specialists through the Oklahoma Medical Research Foundation (OMRF) Sjögren's Research Clinic. Controls were recruited at the OMRF and the University of Michigan. The institutional review boards at the OMRF and the University of Michigan approved this study. All study participants signed a written informed consent form prior to participation.

Isolation and purity of naive CD4+ T cells. Peripheral blood mononuclear cells (PBMCs) were isolated from fresh blood samples obtained from patients and controls using density-gradient centrifugation (Ficoll-Paque; GE Healthcare Life Sciences). Naive CD4+ T cells were separated from PBMCs using a naive CD4+ T Cell Isolation Kit II (Miltenyi Biotec) that allows for indirect isolation of naive CD4 $+\mathrm{T}$ cells, or by sorting the CD3+CD4+CD45RA + naive T cell population on a FACSAria instrument (BD Biosciences). The purity of the naive CD4+ $\mathrm{T}$ cells was confirmed by flow cytometry, using fluorochrome-conjugated antibodies against CD4 and CD45RA. The purity of the isolated naive CD4+ T
Table 1. Demographic characteristics and medication use of the study participants*

\begin{tabular}{|c|c|c|}
\hline Subject/age/sex & Ethnicity & Medication \\
\hline \multicolumn{3}{|c|}{ Patients with primary SS } \\
\hline $1 / 50 /$ female & European American & Methotrexate \\
\hline 2/51/female & European American & Hydroxychloroquine \\
\hline 3/30/female & European American & \\
\hline 4/23/female & European American & Hydroxychloroquine \\
\hline 5/62/female & European American & Azathioprine \\
\hline 6/32/female & European American & \\
\hline 7/35/female & European American & \\
\hline 8/60/female & European American & \\
\hline 9/58/female & European American & \\
\hline 10/35/female & European American & Hydroxychloroquine \\
\hline 11/69/female & European American & Hydroxychloroquine \\
\hline \multicolumn{3}{|l|}{ Healthy controls } \\
\hline 12/52/female & European American & \\
\hline 13/47/female & European American & \\
\hline 14/34/female & European American & \\
\hline $15 / 23 /$ female & European American & \\
\hline 16/58/female & European American & \\
\hline 17/36/female & European American & \\
\hline 18/36/female & European American & \\
\hline 19/55/female & European American & \\
\hline 20/58/female & European American & \\
\hline 21/38/female & European American & \\
\hline 22/64/female & European American & \\
\hline
\end{tabular}

* The mean age of the patients with primary Sjögren's syndrome (SS) and healthy controls was 45.9 years and 45.5 years, respectively $(P=$ $0.95)$.

cells was consistently $>95 \%$, regardless of the method used. DNA was isolated using the DNeasy kit (Qiagen), as described in the manufacturer's protocol.

DNA methylation studies and array validation. Genome-wide DNA methylation in naive CD4+ T cells from the patients with primary SS and healthy controls included in this study was assessed using the Illumina Infinium HumanMethylation450 BeadChip array, which allows for the interrogation of $>485,000$ methylation sites within the entire genome. This array covers $99 \%$ of RefSeq genes, with an average of 17 CpG sites per gene across the promoter region, $5^{\prime}$-untranslated region (5'-UTR), first exon, gene body, and 3 '-UTR. It also covers $96 \%$ of $\mathrm{CpG}$ islands. Non-CpG methylated sites recently identified in human stem cells are also covered, as well as microRNA promoter regions.

Validation of the array data was performed using bisulfite DNA sequencing in selected hypermethylated and hypomethylated gene regions. The primers were as follows: for $H D A C 4$, forward 5'-TGGTTTTATTTTTTGTAGTTAAAAA-3' and reverse 5'-ATAAAACCTCTATACCTCACTCAAC-3'; for $S L C 38 A 4$, forward 5'-TTTGGATTTTTTAATTAAGTTGTTA- $3^{\prime}$ and reverse $5^{\prime}$-TCTACAATATTAATACTCCTACAAACC-3'; for DUSP22, forward 5'-TTATTTGTTTTTTTAGGGTAGGGAG-3' and reverse 5'-AATCTCCAAATCCCCCTTTAAC-3'; for GSTM1, forward 5'-GTTAGGATTTGGTTGGTGTTTTAAG-3' and reverse 5'-ATCCCAATACCCCAATATCATAAAC-3'; for $R U F Y 1$, forward 5'-GTAGGAGAGGTTTTGAGTTGGATT-3' and reverse 5'-TCCTCCATCATCTAАCACTTAAAAA-3'; for NAPRT1, forward 
Table 2. Clinical characteristics of the patients with primary SS $(\mathrm{n}=11)^{*}$

\begin{tabular}{lc}
\hline Dry eyes, no. (\%) & $11(100)$ \\
Dry mouth, no. (\%) & $11(100)$ \\
Schirmer's test for eye dryness & \\
Abnormal findings, no. (\%) & $6(55)$ \\
Mean response, mm & 10.8 \\
Abnormal lissamine green ocular staining score, no. $(\%)$ & $9(82)$ \\
Abnormal WUSF, no. (\%) & $5(45)$ \\
Positive for anti-Ro/SSA, no. (\%) & $6(55)$ \\
Positive for anti-La/SSB, no. (\%) & $6(55)$ \\
MSG biopsy findings consistent with primary SS, no. $(\%)$ & $10(91)$ \\
Mean focus score & 4.5 \\
\hline
\end{tabular}

* The focus score is the number of mononuclear cell infiltrates containing at least 50 inflammatory cells in a $4-\mathrm{mm}^{2}$ glandular section. $\mathrm{SS}=$ Sjögren's syndrome; WUSF = whole unstimulated salivary flow; $\mathrm{MSG}=$ minor salivary gland

5'-TATGGTGGTTTGGTAGAGGTTAGTG-3' and reverse 5'-ACTAATCTATCCTCCACCCTTTCC-3'; and for SLC9A1, forward 5'-GTTTTTTTATTTAGAGAGGGGTAGG-3' and reverse 5'-AACCAAAAAAAACTACAACTAAACC-3'. We used a Bio-Rad T100 Thermocycler with the following protocol: 1 cycle at $94^{\circ} \mathrm{C}$ for 5 minutes, followed by 40 cycles $\left(94^{\circ} \mathrm{C}\right.$ for 45 seconds, annealing temperature for each primer set for 45 seconds, $72^{\circ} \mathrm{C}$ for 90 seconds), and then $74^{\circ} \mathrm{C}$ for 10 minutes. The annealing temperatures for $H D A C 4$, SLC38A, DUSP22, GSTM1, RUFY1, NAPRT1, and SLC9A1 were $54^{\circ} \mathrm{C}, 57^{\circ} \mathrm{C}, 53^{\circ} \mathrm{C}, 54^{\circ} \mathrm{C}, 56^{\circ} \mathrm{C}, 56^{\circ} \mathrm{C}$, and $53^{\circ} \mathrm{C}$, respectively.

We confirmed the presence of polymerase chain reaction (PCR) product with the use of $1.6 \%$ agarose gel electrophoresis. DNA was purified using a QIAquick PCR Purification Kit (Qiagen), as specified by the manufacturer. Bisulfite-treated DNA was sequenced using Sanger sequencing. The DNA methylation level on each $\mathrm{CpG}$ following bisulfite sequencing was quantified using the Epigenomics ESME software package.

Statistical and bioinformatics analyses. DNA methylation studies were performed using the GenomeStudio methylation analysis package (Illumina), as previously described (8). The average level of DNA methylation ( $\beta$ value) on each $\mathrm{CpG}$ site was compared between patients with primary SS and healthy controls. To identify differentially methylated $\mathrm{CpG}$ sites between patients and controls, we used 3 data-filtering criteria: 1) $\mathrm{CpG}$ site with a mean difference in the DNA methylation level of at least 1.2-fold, 2) differential methylation score of $\geq 22(P \leq 0.01)$, after adjustment for multiple testing using a false discovery rate (FDR) of $5 \%$, and 3 ) exclusion of $\mathrm{CpG}$ sites assessed by probes with a genetic variant located within $10 \mathrm{bp}$ of the $3^{\prime}$ end of the probe.

To systematically highlight the most overrepresented biologic terms among the differentially methylated genes, we performed gene ontology (GO), network, and pathway analyses using the DAVID bioinformatics database (version 6.7) (11). In pathway and GO analyses, we used an Expression Analysis Systematic Explorer (EASE) score threshold of $<0.1$ for detection of gene enrichment. The EASE score represents a modified $P$ value determined by Fisher's exact test, which is considered to represent the significance of gene-term enrich- ment. In addition, we used a fold enrichment threshold of 1.5, with an FDR of $<10 \%$ to correct for multiple testing (12).

\section{RESULTS}

We evaluated DNA methylation changes in naive CD4+ T cells from patients with primary SS and age-, sex-, and ethnicity-matched healthy controls. We identified 753 differentially methylated $\mathrm{CpG}$ sites in naive CD4+ T cells from patients with primary SS (Table 3 and Supplementary Table 1, available on the Arthritis \& Rheumatology web site at http://onlinelibrary.wiley.com/ doi/10.1002/art.38264/abstract). Two hundred sites were hypermethylated and 553 were hypomethylated in patients with primary SS as compared to healthy controls. A total of 426 differentially methylated unique genes were identified in naive CD4 $+\mathrm{T}$ cells from patients with primary SS, with the majority of the genes (311 [75\%]) being hypomethylated. Bisulfite DNA sequencing was used to validate the DNA methylation array results by studying a group of hypermethylated (4 genes, 7 loci) and hypomethylated (3 genes, 3 loci) $\mathrm{CpG}$ sites. The mean Pearson's correlation coefficient $\left(\mathrm{r}^{2}\right.$ value) for the correlation between the Illumina Infinium HumanMethylation 450 array data and the bisulfite sequencing data was 0.803 (see Supplementary Figure 1, available on the Arthritis \& Rheumatology web site at http:// onlinelibrary.wiley.com/doi/10.1002/art.38264/abstract).

Although the purity of the naive CD4 $+\mathrm{T}$ cells was consistently $>95 \%$, we wanted to ensure that the differential methylation patterns identified between patients and controls in our study were not influenced by potential differences in the naive $\mathrm{CD} 4+\mathrm{T}$ cell activation status, which could have been induced by the different isolation procedures used. We therefore examined the DNA methylation levels of a region in the IL2 promoter-enhancer sequence that is known to readily demethylate upon $\mathrm{T}$ cell activation. Demethylation of

Table 3. Summary of the differentially methylated $\mathrm{CpG}$ sites and genes in naive $\mathrm{CD} 4+\mathrm{T}$ cells from patients with primary Sjögren's syndrome as compared to healthy controls

\begin{tabular}{lccc}
\hline & $\begin{array}{c}\text { Increased } \\
\text { methylation }\end{array}$ & $\begin{array}{c}\text { Decreased } \\
\text { methylation }\end{array}$ & Total \\
\hline $\begin{array}{l}\text { No. of CpG sites } \\
\text { Fold change }\end{array}$ & 200 & 553 & 753 \\
$\quad$ Range & $1.20,5.20$ & $1.20,5.22$ & \\
$\quad$ Mean & 1.65 & 1.60 & \\
Differential score & & & \\
$\quad$ Range & $22.0,342.0$ & $-22.0,-339.8$ & \\
$\quad$ Mean & +95.6 & -66.1 & 426 \\
No. of genes & 115 & 311 & 426 \\
\hline
\end{tabular}




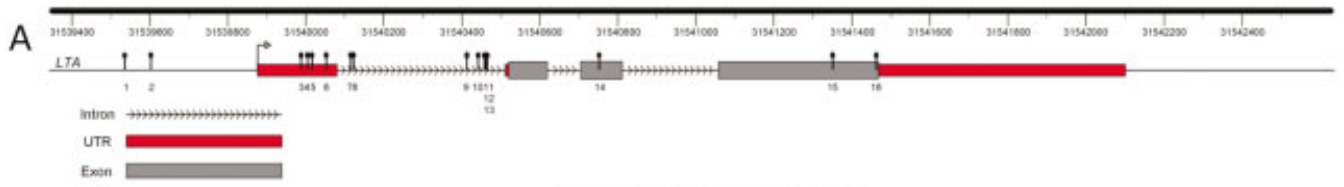

\section{LTA Methylation}

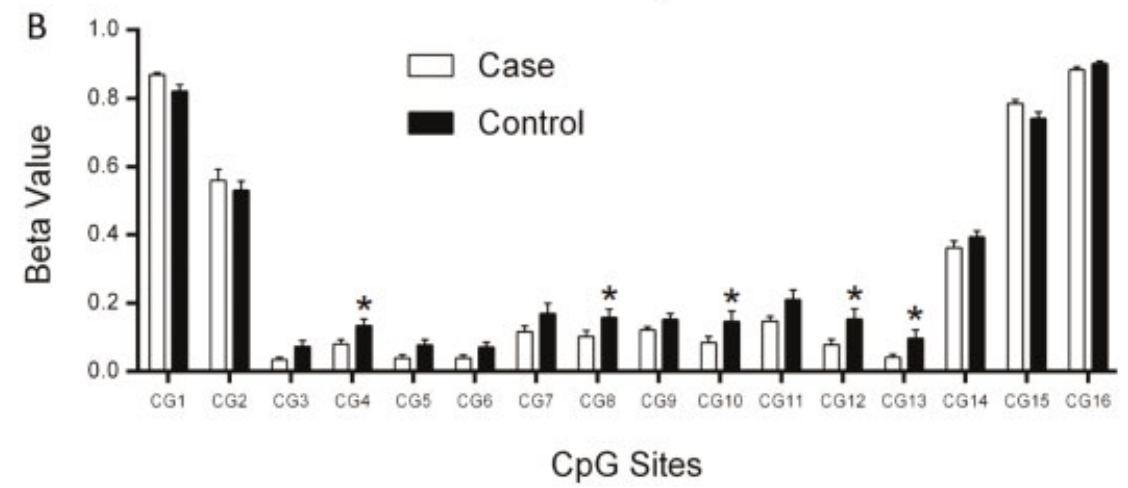

Figure 1. DNA methylation of the LTA gene (encoding lymphotoxin $\alpha$ ) in naive CD4+ T cells from patients with primary Sjögren's syndrome and healthy controls. A, Schematic of $L T A$ DNA methylation (31,539,814-31,542,537 bp on chromosome 6; GRCh37/hg19), depicting the location of the $\mathrm{CpG}$ sites evaluated. UTR $=$ untranslated region. B, DNA methylation fractions ( $\beta$ values) across the CpG sites in $L T A$, evaluated in naive CD4+ $\mathrm{T}$ cells from patients with primary Sjögren's syndrome and healthy controls. Asterisks indicate the 5 hypomethylated CpG sites in the $L T A$ gene region in cases compared to controls. Values are the mean $\pm \mathrm{SD}$.

the promoter-enhancer region of $I L 2$ is a sensitive indicator of $\mathrm{CD} 4+\mathrm{T}$ cell activation $(13,14)$ and is a prerequisite for $I L 2$ transcription (15). We detected high DNA methylation levels in the IL2 promoter-enhancer region, as expected in naive CD4+ T cells, and there was no difference between patients and controls (see Supplementary Table 2, available on the Arthritis \& Rheumatology web site at http://onlinelibrary.wiley.com/doi/ 10.1002/art.38264/abstract).

Furthermore, we examined the DNA methylation levels in genetic loci known to demethylate in specific antigen-experienced and regulatory $\mathrm{CD} 4+\mathrm{T}$ cell subsets, including IFNG (Th1 cells), IL4, IL5, and IL13 (Th2 cells), IL17 (IL17A) and IL17F (Th17 cells), and FOXP3 (regulatory T cells). Again, we observed that the DNA methylation levels were consistently high across these genetic loci, and there was no difference between patients and controls (see Supplementary Table 2, available on the Arthritis \& Rheumatology web site at http:// onlinelibrary.wiley.com/doi/10.1002/art.38264/abstract).

The hypomethylated genes detected in naive CD4 $+\mathrm{T}$ cells from patients with primary SS included $L T A$ (encoding lymphotoxin $\alpha[\mathrm{LT} \alpha]$ ), which is involved in the LT $\beta$ receptor (LT $\beta \mathrm{R}$ ) signaling pathway, activation of follicular dendritic cells, and expression of IFN $\alpha$. We identified 5 differentially methylated $\mathrm{CpG}$ sites in LTA, located at the 5'-UTR within 200 bp upstream of the transcriptional start site (hereafter referred to as TSS 200) and in the first exon (Figure 1). The mean differential methylation score (Diff. score) of the $5 \mathrm{CpG}$ sites was -35.4 (range -22.3 to -53.8 ; mean fold change 1.82). Other relevant hypomethylated genes included CD247, which encodes for T cell receptor (TCR) $\zeta$-chain $(2 \mathrm{CpG}$ sites located in the body of the gene, with Diff. scores of -23.9 and -32.3 , respectively; mean fold change 1.96), TNFRSF25 (1 CpG site located 1,500 bp upstream of the TSS [TSS 1,500], with a Diff. score of -23.2; mean fold change 1.59), PTPRC (2 CpG sites in the body of the gene and in TSS 1,500, with Diff. scores of -49.7 and -75.5 , respectively; mean fold change 1.96), GSTM1 (2 CpG sites located in TSS 200, with Diff. scores of -31.4 and -48.8 , respectively; mean fold change 1.40), and PDCD1 (1 CpG site located in TSS 1,500 , with a Diff. score of -46.9 ; mean fold change 2.63). The type I IFN pathway, which plays a major role in the pathogenesis of primary SS (16), was represented by hypomethylation of STAT1 (2 CpG sites located in the 5'-UTR, with Diff. scores of -23.9 and -79.2 , respectively; mean fold change 1.41), IFI44L (1 CpG site located in the $5^{\prime}$-UTR, with a Diff. score of -24.1; mean fold change 1.22), IFITM1 (1 CpG site located in TSS 1,500 , with a Diff. score of -35.8 ; mean fold change 1.75), and USP18 (1 CpG site located in the 5'-UTR, with a Diff. score of -65.1 ; mean fold change 1.40 ). 
Table 4. Pathway analysis of the differentially methylated genes in naive CD4+ T cells from patients with primary Sjögren's syndrome as compared to healthy controls*

\begin{tabular}{|c|c|c|c|c|c|}
\hline Category & Term & Molecule & $P$ & $\begin{array}{c}\text { Fold } \\
\text { enrichment }\end{array}$ & FDR \\
\hline KEGG_PATHWAY & Type 1 diabetes mellitus & $\begin{array}{l}\text { PTPRN2; CD28; LTA; HLA-A; HLA-B; } \\
\text { HLA-C; HLA-F; HLA-DMA; HLA-DRB1; } \\
\text { HLA-DRB4; HLA-DRB5 }\end{array}$ & $4.59 \times 10^{-6}$ & 9.005 & 0.005 \\
\hline KEGG_PATHWAY & Allograft rejection & $\begin{array}{l}\text { CD28; HLA-A; HLA-B; HLA-C; HLA-F; } \\
\text { HLA-DMA; HLA-DRB1; HLA-DRB4; } \\
\text { HLA-DRB5 }\end{array}$ & $1.66 \times 10^{-4}$ & 8.171 & 0.191 \\
\hline KEGG_PATHWAY & Viral myocarditis & $\begin{array}{l}\text { MYH13; CD28; CASP9; HLA-A; HLA-B; } \\
\text { HLA-C; HLA-F; HLA-DMA; HLA-DRB1; } \\
\text { HLA-DRB4; HLA-DRB5 }\end{array}$ & $2.34 \times 10^{-4}$ & 5.327 & 0.268 \\
\hline KEGG_PATHWAY & Graft-versus-host disease & $\begin{array}{l}\text { CD28; HLA-A; HLA-B; HLA-C; HLA-F; } \\
\text { HLA-DMA; HLA-DRB1; HLA-DRB4; } \\
\text { HLA-DRB5 }\end{array}$ & $2.63 \times 10^{-4}$ & 7.543 & 0.301 \\
\hline KEGG_PATHWAY & Autoimmune thyroid disease & $\begin{array}{l}\text { CD28; HLA-A; HLA-B; HLA-C; HLA-F; } \\
\text { HLA-DMA; HLA-DRB1; HLA-DRB4; } \\
\text { HLA-DRB5 }\end{array}$ & $1.15 \times 10^{-3}$ & 5.768 & 1.315 \\
\hline KEGG_PATHWAY & Antigen processing and presentation & $\begin{array}{l}\text { LTA; HLA-A; HLA-B; HLA-C; HLA-F; } \\
\text { HLA-DMA; HLA-DRB1; HLA-DRB4; } \\
\text { HLA-DRB5; TAP2 }\end{array}$ & $3.22 \times 10^{-3}$ & 4.051 & 3.637 \\
\hline KEGG_PATHWAY & Cell adhesion molecules & $\begin{array}{l}\text { NCAM1; SELL; PDCD1; CD28; HLA-A; } \\
\text { HLA-B; HLA-C; HLA-F; HLA-DMA; } \\
\text { HLA-DRB1; HLA-DRB4; HLA-DRB5 }\end{array}$ & $3.73 \times 10^{-3}$ & 3.184 & 4.201 \\
\hline
\end{tabular}

${ }^{*} \mathrm{FDR}=$ false discovery rate; KEGG $=$ Kyoto Encyclopedia of Genes and Genomes.

Table 5. GO analysis of hypomethylated and hypermethylated genes in naive CD4+ T cells from patients with primary Sjögren's syndrome*

\begin{tabular}{|c|c|c|c|c|c|c|}
\hline Category & GO term & $\begin{array}{l}\text { GO } \\
\text { identification } \\
\text { no. }\end{array}$ & Gene & $P$ & $\begin{array}{l}\text { Fold } \\
\text { enrichment }\end{array}$ & FDR \\
\hline \multicolumn{7}{|l|}{ Hypomethylated genes } \\
\hline GOTERM_BP_FAT & Lymphocyte activation & 0046649 & $\begin{array}{l}\text { BCL11B; CD3G; CD28; CD7; CHD7; } \\
\text { FOXP1; HDAC4; ITPKB; JMJD6; } \\
\text { RHOH; SLC11A1; SKAP2; UNC13D }\end{array}$ & $1.10 \times 10^{-4}$ & 3.859 & 0.185 \\
\hline GOTERM_BP_FAT & Leukocyte differentiation & 0002521 & $\begin{array}{l}\text { BCL11B; CEBPE; CD28; CHD7; } \\
\text { FOXP1; HDAC4; ITPKB; JMJD6; } \\
\text { RHOH }\end{array}$ & $1.42 \times 10^{-3}$ & 4.168 & 2.361 \\
\hline GOTERM_BP_FAT & Immune response & 0006955 & $\begin{array}{l}\text { BTLA; CD28; CD300A; CD7; FAIM3; } \\
\text { ST6GAL1; C4BPB; FOXP1; IFI44L; } \\
\text { IL36G; IL16; LY86; LTA; HLA-A; } \\
\text { HLA-B; HLA-H; HLA-DRB5; } \\
\text { PDCD1; SLC11A1; TCF12; TCF7; } \\
\text { UNC13D; ETS1 }\end{array}$ & $2.20 \times 10^{-3}$ & 1.969 & 3.646 \\
\hline GOTERM_BP_FAT & Chromatin organization & 0006325 & $\begin{array}{l}\text { BCOR; CREBBP; DNMT3A; H2AFV; } \\
\text { SATB1; SMARCB1; APBB1; CHD7; } \\
\text { HDAC4; JMJD6; KDM2B; RBBP7; } \\
\text { TSPY4; TSPY1; TSPY2; TSPY3; } \\
\text { TSPY7P; TLK1 }\end{array}$ & $3.90 \times 10^{-3}$ & 2.344 & 6.356 \\
\hline GOTERM_BP_FAT & $\mathrm{T}$ cell differentiation & 0030217 & $\begin{array}{l}\text { BCL11B; CD28; CHD7; ITPKB; } \\
\text { JMJD6; RHOH }\end{array}$ & $4.20 \times 10^{-3}$ & 5.453 & 6.855 \\
\hline GOTERM_BP_FAT & Homophilic cell adhesion & 0007156 & $\begin{array}{l}\text { PCDHAC2; PCDHA2; PCDHA3; } \\
\text { PCDHA4; PCDHA5; PCDHA7; } \\
\text { PCDHA8; PCDHA9 }\end{array}$ & $5.80 \times 10^{-3}$ & 3.608 & 9.360 \\
\hline GOTERM_BP_FAT & L-amino acid transport & 0015807 & CACNA1A; HTT; SLC11A1; SLC7A8 & $6.10 \times 10^{-3}$ & 10.274 & 9.687 \\
\hline \multicolumn{7}{|l|}{ Hypermethylated genes } \\
\hline GOTERM_BP_FAT & $\begin{array}{l}\text { Antigen processing and } \\
\text { presentation }\end{array}$ & 0019882 & $\begin{array}{l}\text { TAP2; HLA-B; HLA-C; HLA-F; } \\
\text { HLA-H; HLA-DMA; HLA-DRB4; } \\
\text { HLA-DRB5 }\end{array}$ & $6.89 \times 10^{-6}$ & 14.817 & 0.010 \\
\hline
\end{tabular}

\footnotetext{
* Statistically significant gene ontology $(\mathrm{GO})$ terms are shown. FDR $=$ false discovery rate; GOTERM_BP_FAT = gene ontology term biologic process.
} 
A group of genes encoding members of the solute carrier proteins, which are membrane transport proteins that are important for maintenance of cell function, were hypomethylated, including SLC11A1, SLC11A2, SLC22A23, SLC25A25, SLC25A3, SLC25A33, and $S L C 6 A 20$, whereas $S L C 9 A 1$, which is important for the maintenance of $\mathrm{pH}$ homeostasis, was hypermethylated in patients with primary SS as compared to healthy controls. In addition, the transcription factor RUNX1 was hypermethylated in patients with primary SS. Supplementary Table 1 (available on the Arthritis \& Rheumatology web site at http://onlinelibrary.wiley.com/doi/ 10.1002/art.38264/abstract) provides a summary of all CpG sites that were differentially methylated in patients with primary SS in our study.

Next, we used DAVID software (11) to facilitate the systematic identification and grouping of differentially methylated genes into biologic networks. Canonical pathway analysis identified type 1 diabetes mellitus $\left(P=4.59 \times 10^{-6}\right)$, allograft rejection $\left(P=1.66 \times 10^{-4}\right)$, viral myocarditis $\left(P=2.34 \times 10^{-4}\right)$, graft-versus-host disease $\left(P=2.63 \times 10^{-4}\right)$, autoimmune thyroid disease $\left(P=1.15 \times 10^{-3}\right)$, antigen processing and presentation $\left(P=3.22 \times 10^{-3}\right)$, and cell adhesion molecules $(P=$ $\left.3.73 \times 10^{-3}\right)$ as the most significant pathways unifying the differentially methylated genes in naive CD4 $+\mathrm{T}$ cells from patients with primary SS (Table 4).

GO analysis of hypomethylated genes demonstrated enrichment of genes involved in lymphocyte activation $\left(P=1.10 \times 10^{-4}\right)$, leukocyte differentiation $\left(P=1.42 \times 10^{-3}\right)$, immune response $\left(P=2.20 \times 10^{-3}\right)$, chromatin organization $\left(P=3.90 \times 10^{-3}\right)$, T cell differentiation $\left(P=4.20 \times 10^{-3}\right)$, homophilic cell adhesion $\left(P=5.80 \times 10^{-3}\right)$, and L-amino acid transport $\left(P=6.10 \times 10^{-3}\right)$. GO terms for hypermethylated genes included antigen processing and presentation ontology $\left(P=6.89 \times 10^{-6}\right)($ Table 5).

\section{DISCUSSION}

In this study, we performed an unbiased genomewide DNA methylation analysis of naive CD4 $+\mathrm{T}$ cells from patients with primary SS, and thereby identified a set of differentially methylated genes and involved pathways in patients with primary SS as compared to matched healthy controls. The molecular basis of primary SS is not well characterized; however, cumulative evidence indicates that activation of the LT $\beta$ R pathway plays an integral role in the pathogenesis of primary SS (17). We identified 5 hypomethylated $\mathrm{CpG}$ sites in the
LTA gene in naive CD4+ $\mathrm{T}$ cells from patients with primary SS as compared to healthy controls.

Lymphotoxins (LT $\alpha, \mathrm{LT} \beta)$ and their receptors are part of the tumor necrosis factor superfamily (18). Soluble LT $\alpha$ promotes production of IFNs and multiple chemokines that are important activating signals to the immune cells (19). Moreover, LT $\alpha$ forms a heterodimer with $\operatorname{LT} \beta(\operatorname{LT} \alpha 1 \beta 2)$, which in turn binds to $\operatorname{LT} \beta \mathrm{R}$ (20). Activation of the LT $\beta$ R pathway is crucial for the development, organization, and maintenance of lymphoid structures (21) and for modulating the expression of chemokines and adhesion molecules that aid in trafficking of lymphocytes and follicular dendritic cell activation (22).

Furthermore, studies in murine models have highlighted an interesting role of LT $\alpha$ in the pathogenesis of Sjögren's-like disease. LT $\alpha$ was found to be overexpressed in the salivary gland secretions and sera of IL14 $\alpha$-transgenic (TG) mice, a murine model of SS (23). The IL14 $\alpha$-TG mouse reproduces the clinical and immunologic changes characteristic of SS (24). Interestingly, IL14 $\alpha$-TG mice with deletion of the $L T A$ gene did not develop disease (23). In another murine model of SS using NOD mice, Gatumu and colleagues (22) showed that blocking the LT $\beta$ R pathway resulted in ablation of the lymphoid organization in the NOD mouse salivary glands and an improvement in salivary gland function (22). In another study, blocking of LT $\beta$ R reduced the size of leukocyte infiltrates in the lacrimal glands, and improved tear production and corneal integrity (25). Additional evidence that $\mathrm{LT} \alpha$ has a role in SS comes from the observation that $\mathrm{LT} \alpha$ is overexpressed in the salivary glands and sera of patients with SS (23). Indeed, a clinical trial to evaluate the use of an LT $\beta$ R fusion protein (baminercept) in the treatment of patients with primary SS is currently in progress. Our results suggest that epigenetic factors may play a role in $\mathrm{LT} \alpha$ overexpression in patients with primary SS, as cytosine demethylation predisposes patients to transcriptionally permissive chromatin architecture in this locus.

Patients with primary SS have an activated type I IFN response $(16,26)$, as has been demonstrated by observations of increased type I IFN activity and an "IFN signature" in PBMCs (27), saliva (28), and minor salivary gland biopsy tissue $(29,30)$ from patients with primary SS. Several of the hypomethylated genes that were identified herein in naive $\mathrm{CD} 4+\mathrm{T}$ cells from patients with primary SS were IFN-regulated genes (STAT1, IFI44L, IFITM1, and USP18). The extent of hypomethylation of IFN-regulated genes seems to be less robust in patients with primary SS in comparison to 
patients with SLE (9), in whom we have recently identified and reproduced 21 hypomethylated IFN-regulated genes in naive $\mathrm{CD} 4+\mathrm{T}$ cells.

In this study, we identified several differentially methylated genes that are important in activation of the immune system. CD247 encodes for the TCR $\zeta$-chain, is important for signal transduction upon antigen stimulation (31), and was hypomethylated in naive CD4 $+\mathrm{T}$ cells in patients with primary SS. Other pertinent genes included PTPRC, which encodes protein tyrosine phosphatase, receptor type C (also known as CD45 antigen or B220). PTPRC is a signaling molecule that is essential for $\mathrm{T}$ and $\mathrm{B}$ cell activation, cellular differentiation, and oncogenic transformation (32). TNFRSF25, which plays a role in lymphocyte homeostasis and in apoptosis, was also hypomethylated in patients with primary SS. Furthermore, we detected hypomethylation of GSTM1, which encodes a cytoplasmic glutathione S-transferase that is important in detoxification of electrophilic compounds, including carcinogens and environmental toxins (33). Of particular interest, GSTM1 was proposed as a genetic risk locus for primary SS in a previous study (34), which might suggest that this gene has a role in geneticepigenetic interactions in the pathogenesis of primary SS. Indeed, we have previously demonstrated a genetic association between primary SS and variants in MECP2 (encoding methyl-CpG binding protein 2), a key transcriptional regulator with a critical role in DNA methylation (35). Among the transcription factors, we identified $R U N X 1$ as a hypermethylated gene in the present study. RUNX-1 regulates the differentiation of hematopoietic stem cells into mature cells (36) and has been linked to cancer predisposition $(37,38)$. These findings suggest that $R U N X 1$ has a possible connection to lymphoma predisposition in primary SS.

It is generally accepted that defects in membrane water channel proteins contribute to the exocrinopathy in patients with primary SS (39). Our study identified several differentially methylated genes encoding members of the solute carrier proteins. The genes SLC11A1, SLC11A2, SLC22A23, SLC25A25, SLC25A3, $S L C 25 A 33$, and $S L C 6 A 20$ were hypomethylated, whereas $S L C 9 A 1$, which is expressed in the kidneys and plays an important role in the maintenance of $\mathrm{pH}$ homeostasis, was hypermethylated in patients with primary SS as compared to healthy controls. Mutations in solute carrier proteins have been previously linked to diseases associated with acid-base disturbances, such as Bartter and Gitelman syndromes (40). We hypothesize that methylation changes in these proteins may explain some of the pathologic aspects of primary SS-like defects in molecular water transport, dysfunction of the exocrine glands, and perhaps distal renal tubular acidosis in some patients with primary SS (41).

We used the DAVID database to facilitate the systematic identification and grouping of differentially methylated genes into biologic networks. Of particular interest, there was enrichment of ontologies involved in both the adaptive and the innate immune systems, underscoring involvement of the 2 arms of immunity in the pathogenesis of primary SS (Table 5).

Two cell-separation methods were used to isolate naive $\mathrm{CD} 4+\mathrm{T}$ cells from patients and controls, albeit with consistent and equal cell purity $(>95 \%)$. We further confirmed the absence of $\mathrm{T}$ cell activation or differentiation in the study samples with the use of epigenetic immunophenotyping, in which we examined the methylation status of IL2, IFNG, IL4, IL5, IL13, $I L 17, I L 17 F$, and FOXP3. It is important to note that identification of the phenotypic specificity of cells is critical to accurately and reliably interpret differential methylation data in autoimmune diseases, while avoiding any perceived differences that could be related to altered cell constituents.

DNA methylation is tightly linked to chromatin accessibility, and gene expression requires both chromatin accessibility and appropriate transcription factors. Therefore, some, but not all, of the differences in DNA methylation identified in the present study can be expected to be associated with expression differences. Examining DNA methylation at a genome-wide level provides another level for discovering fundamental differences that might be pathogenic in the disease process at the chromatin level, which might or might not be linked directly to expression differences. For example, we recently observed that naive CD4 $+\mathrm{T}$ cells from lupus patients had widespread hypomethylation of IFNregulated genes, before an expression difference could be detected (9). Interestingly, to our knowledge, there has not yet been a gene expression study of naive CD4+ $\mathrm{T}$ cells from patients with primary SS, and therefore we do not know which genes are differentially expressed in naive $\mathrm{CD} 4+\mathrm{T}$ cells in this disease. Therefore, whether some of the methylation changes detected in this first DNA methylation study in patients with primary SS are reflective of a difference in the active (or potential) gene expression state remains to be seen.

The recent advances in epigenetics and the emergence of epigenome-wide association studies have resulted in the successful identification of numerous intriguing associations between epigenomic perturbations and human disease. However, this field is still in its 
infancy and the issue of "causality" related to the detected epigenetic changes will require careful experimental examination. The issue of causality inference is even more complicated in patients with primary SS, for several reasons. First, understanding of the genetic predisposition to primary SS, which might influence epigenetic variations, is incomplete. Second, the environmental factors involved in the pathogenesis of primary SS remain, by and large, uncharacterized. Third, our study was designed as a retrospective case-control study; alternative study designs would be longitudinal studies of monozygotic twins discordant for primary SS or prospective studies to evaluate epigenetic perturbations prior to the onset of the disease, both of which are study designs that are cumbersome for obvious reasons. Nevertheless, such studies are warranted in the future to move this field forward (42).

Integrating emerging genomic data from patients with primary SS with epigenomic profiling might provide an avenue to discover mechanistic and pathogenic pathways to this disease. For example, we suspect that some genetic susceptibility variants that will be discovered in patients with primary SS might influence some of the methylation differences observed. A simultaneous genomic-epigenomic analysis should therefore focus on allele-specific methylation changes induced by genetic variants associated with primary SS. These approaches will help to identify novel mechanisms and therapeutic targets for this disease, and might enhance our understanding of the functional consequences of some of the genetic susceptibility loci in primary SS.

In summary, we identified, for the first time, DNA methylation changes in naive $\mathrm{CD} 4+\mathrm{T}$ cells from patients with primary SS. These data indicate that abnormal DNA methylation exists in CD4 $+\mathrm{T}$ cells, even before the activation and differentiation of $\mathrm{T}$ cells, in patients with primary SS. Therefore, our findings emphasize the potential role of DNA methylation changes in the pathogenesis of primary SS. Our study demonstrates differential methylation of LTA, type I IFN-regulated genes, and solute carrier proteins, in addition to other key genes and pathways involved in the pathogenesis of primary SS. Future studies to replicate and determine the functional consequences of the methylation changes observed upon pathophysiologic development of the disease are warranted.

\section{ACKNOWLEDGMENTS}

We are grateful to the clinicians and staff of the OMRF Sjögren's Research Clinic (Kim Hefner, David Lewis, and Glen Houston). We also thank Christina Lawrence (OMRF) for her expert technical assistance.

\section{AUTHOR CONTRIBUTIONS}

All authors were involved in drafting the article or revising it critically for important intellectual content, and all authors approved the final version to be published. Dr. Sawalha had full access to all of the data in the study and takes responsibility for the integrity of the data and the accuracy of the data analysis.

Study conception and design. Altorok, Scofield, Farris, Sawalha. Acquisition of data. Altorok, Coit, Hughes, Stone, Rasmussen, Radfar, Scofield, Sivils, Farris, Sawalha.

Analysis and interpretation of data. Altorok, Hughes, Koelsch, Sawalha.

\section{REFERENCES}

1. Fox RI. Sjögren's syndrome. Lancet 2005;366:321-31.

2. Delaleu N, Jonsson R, Koller MM. Sjögren's syndrome. Eur J Oral Sci 2005;113:101-13.

3. Hughes GR, Whaley K. Sjögren's syndrome. Br Med J 1972;4: 533-6.

4. Kassan SS, Thomas TL, Moutsopoulos HM, Hoover R, Kimberly $\mathrm{RP}$, Budman DR, et al. Increased risk of lymphoma in sicca syndrome. Ann Intern Med 1978;89:888-92.

5. Kassan SS, Moutsopoulos HM. Clinical manifestations and early diagnosis of Sjögren syndrome. Arch Intern Med 2004;164: 1275-84.

6. Altorok N, Sawalha AH. Epigenetics in the pathogenesis of systemic lupus erythematosus. Curr Opin Rheumatol 2013;25: 569-76.

7. Thabet Y, Le Dantec C, Ghedira I, Devauchelle V, Cornec D, Pers $\mathrm{JO}$, et al. Epigenetic dysregulation in salivary glands from patients with primary Sjögren's syndrome may be ascribed to infiltrating B cells. J Autoimmun 2013;41:175-81.

8. Jeffries MA, Sawalha AH. Epigenetics in systemic lupus erythematosus: leading the way for specific therapeutic agents. Int $\mathbf{J}$ Clin Rheumatol 2011;6:423-39.

9. Coit P, Jeffries M, Altorok N, Dozmorov MG, Koelsch KA, Wren JD, et al. Genome-wide DNA methylation study suggests epigenetic accessibility and transcriptional poising of interferonregulated genes in naive $\mathrm{CD} 4+\mathrm{T}$ cells from lupus patients. J Autoimmun 2013;43:78-84.

10. Vitali C, Bombardieri S, Jonsson R, Moutsopoulos HM, Alexander EL, Carsons SE, et al, and the European Study Group on Classification Criteria for Sjögren's Syndrome. Classification criteria for Sjögren's syndrome: a revised version of the European criteria proposed by the American-European Consensus Group. Ann Rheum Dis 2002;61:554-8.

11. Dennis G Jr, Sherman BT, Hosack DA, Yang J, Gao W, Lane HC, et al. DAVID: Database for Annotation, Visualization, and Integrated Discovery. Genome Biol 2003;4:P3.

12. Huang DW, Sherman BT, Lempicki RA. Systematic and integrative analysis of large gene lists using DAVID bioinformatics resources. Nat Protoc 2009;4:44-57.

13. Bruniquel D, Schwartz RH. Selective, stable demethylation of the interleukin-2 gene enhances transcription by an active process. Nat Immunol 2003;4:235-40.

14. Bird A. Il2 transcription unleashed by active DNA demethylation. Nat Immunol 2003;4:208-9.

15. Murayama A, Sakura K, Nakama M, Yasuzawa-Tanaka K, Fujita $\mathrm{E}$, Tateishi $\mathrm{Y}$, et al. A specific $\mathrm{CpG}$ site demethylation in the human interleukin 2 gene promoter is an epigenetic memory. EMBO J 2006;25:1081-92.

16. Mavragani CP, Crow MK. Activation of the type I interferon 
pathway in primary Sjögren's syndrome. J Autoimmun 2010;35: 225-31.

17. Rennert PD, James D, Mackay F, Browning JL, Hochman PS. Lymph node genesis is induced by signaling through the lymphotoxin $\beta$ receptor. Immunity 1998;9:71-9.

18. Fava RA, Browning JL, Gatumu M, Skarstein K, Bolstad AI LTBR-pathway in Sjögren's syndrome: CXCL13 levels and B-cellenriched ectopic lymphoid aggregates in NOD mouse lacrimal glands are dependent on LTBR. Adv Exp Med Biol 2011;691: 383-90.

19. Ware CF. Network communications: lymphotoxins, LIGHT, and TNF. Annu Rev Immunol 2005;23:787-819.

20. Browning JL, Dougas I, Ngam-ek A, Bourdon PR, Ehrenfels BN, Miatkowski K, et al. Characterization of surface lymphotoxin forms: use of specific monoclonal antibodies and soluble receptors. J Immunol 1995; 154:33-46.

21. Fu YX, Chaplin DD. Development and maturation of secondary lymphoid tissues. Annu Rev Immunol 1999;17:399-433.

22. Gatumu MK, Skarstein K, Papandile A, Browning JL, Fava RA, Bolstad AI. Blockade of lymphotoxin- $\beta$ receptor signaling reduces aspects of Sjögren's syndrome in salivary glands of non-obese diabetic mice. Arthritis Res Ther 2009;11:R24.

23. Shen L, Suresh L, Wu J, Xuan J, Li H, Zhang C, et al. A role for lymphotoxin in primary Sjögren's disease. J Immunol 2010;185: 6355-63.

24. Shen L, Suresh L, Li H, Zhang C, Kumar V, Pankewycz O, et al. IL-14 $\alpha$, the nexus for primary Sjögren's disease in mice and humans. Clin Immunol 2009;130:304-12.

25. Fava RA, Kennedy SM, Wood SG, Bolstad AI, Bienkowska J, Papandile A, et al. Lymphotoxin- $\beta$ receptor blockade reduces CXCL13 in lacrimal glands and improves corneal integrity in the NOD model of Sjögren's syndrome. Arthritis Res Ther 2011;13: R182.

26. Bave U, Nordmark G, Lovgren T, Ronnelid J, Cajander S, Eloranta ML, et al. Activation of the type I interferon system in primary Sjögren's syndrome: a possible etiopathogenic mechanism. Arthritis Rheum 2005;52:1185-95.

27. Emamian ES, Leon JM, Lessard CJ, Grandits M, Baechler EC, Gaffney PM, et al. Peripheral blood gene expression profiling in Sjögren's syndrome. Genes Immun 2009;10:285-96.

28. Hu S, Wang J, Meijer J, Ieong S, Xie Y, Yu T, et al. Salivary proteomic and genomic biomarkers for primary Sjögren's syndrome. Arthritis Rheum 2007;56:3588-600.

29. Hjelmervik TO, Petersen K, Jonassen I, Jonsson R, Bolstad AI. Gene expression profiling of minor salivary glands clearly distinguishes primary Sjögren's syndrome patients from healthy control subjects. Arthritis Rheum 2005;52:1534-44.
30. Gottenberg JE, Cagnard N, Lucchesi C, Letourneur F, Mistou S, Lazure $\mathrm{T}$, et al. Activation of IFN pathways and plasmacytoid dendritic cell recruitment in target organs of primary Sjögren's syndrome. Proc Natl Acad Sci U S A 2006;103:2770-5.

31. Weissman AM, Hou D, Orloff DG, Modi WS, Seuanez H, O'Brien SJ, et al. Molecular cloning and chromosomal localization of the human T-cell receptor $\zeta$ chain: distinction from the molecular CD3 complex. Proc Natl Acad Sci U S A 1988;85:9709-13.

32. Clark MC, Pang M, Hsu DK, Liu FT, de Vos S, Gascoyne RD, et al. Galectin-3 binds to CD45 on diffuse large B-cell lymphoma cells to regulate susceptibility to cell death. Blood 2012;120: 4635-44.

33. Sun ZF, Zhang J, Xu HM, Wang GL, Dong P. Association between GSTM1 polymorphism and nasopharyngeal cancer susceptibility: a meta-analysis. Asian Pac J Cancer Prev 2012;13 5817-21.

34. Morinobu A, Kanagawa S, Koshiba M, Sugai S, Kumagai S. Association of the glutathione S-transferase M1 homozygous nul genotype with susceptibility to Sjögren's syndrome in Japanese individuals. Arthritis Rheum 1999;42:2612-5.

35. Cobb BL, Fei Y, Jonsson R, Bolstad AI, Brun JG, Rischmueller $\mathrm{M}$, et al. Genetic association between methyl-CpG binding protein 2 (MECP2) and primary Sjögren's syndrome. Ann Rheum Dis 2010;69:1731-2.

36. Okuda T, Nishimura M, Nakao M, Fujita Y. RUNX1/AML1: central player in hematopoiesis. Int J Hematol 2001;74:252-7.

37. Asou N. The role of a Runt domain transcription factor AML1/ RUNX1 in leukemogenesis and its clinical implications [published erratum appears in Crit Rev Oncol Hematol 2006;60:87]. Crit Rev Oncol Hematol 2003;45:129-50.

38. Kundu M, Compton S, Garrett-Beal L, Stacy T, Starost MF, Eckhaus $\mathrm{M}$, et al. Runx1 deficiency predisposes mice to $\mathrm{T}$ lymphoblastic lymphoma. Blood 2005;106:3621-4.

39. Nikolov NP, Illei GG. Pathogenesis of Sjögren's syndrome. Curr Opin Rheumatol 2009;21:465-70.

40. Hediger MA, Romero MF, Peng JB, Rolfs A, Takanaga H, Bruford EA. The ABCs of solute carriers: physiological, pathological and therapeutic implications of human membrane transport proteins: introduction. Pflugers Arch 2004;447:465-8.

41. Pertovaara M, Korpela M, Kouri T, Pasternack A. The occurrence of renal involvement in primary Sjögren's syndrome: a study of 78 patients. Rheumatology (Oxford) 1999;38:1113-20.

42. Relton CL, Davey Smith G. Two-step epigenetic Mendelian randomization: a strategy for establishing the causal role of epigenetic processes in pathways to disease. Int J Epidemiol 2012;41: $161-76$. 